Journal of Mathematics and Statistics 5 (4): 270-275, 2009

ISSN 1549-3644

(C) 2009 Science Publications

\title{
Random Sum of Mixtures of Sum of Bivariate Exponential Distributions
}

\author{
Abd El-Moneim A.M. Teamah and Ahmed M.T. Abd El-Bar \\ Department of Mathematics, Faculty of Science, Tanta University, Tanta, Egypt
}

\begin{abstract}
Problem statement: The distributions of $R=X+Y$ and $W=X /(X+Y)$, where $X$ and $Y$ follow Lawrance and Lewis`s bivariate exponential distribution, is generalized. Approach: $I n$ this research we found mixtures of sum of bivariate exponential random variables. Results: Also we calculated the probability density function (pdf) of the random sum of mixtures of sum of bivariate exponential random variables. Conclusion/Recommendations: In this study we investigated the pdf of random sum of mixtures of sum of bivariate exponential random variables.
\end{abstract}

Key words: Lawrance and Lewis`s bivariate exponential distribution, random sum, mixture distribution, Laplace transform

\section{INTRODUCTION}

One of the standard stochastic models used in various areas of applied probability, such as insurance risk theory and queuing theory, is the random sum model. Random sum is defined as a sum of $n$ random variables and the number $n$ is also random variable. Random sum play an important role in insurance application, for example, let $X_{i}, i=1,2, \ldots, N$ be independent and identically distributed random variables determine the losses of a unit of production caused by fire, explosion, destruction, and so on. The random sum of $X_{i}, i=1,2, \ldots, N$ is the total losses of this unit, and then one can get tail probability, i.e., $\quad a_{t}=\operatorname{Pr}\left(S_{N}>t\right)$. Compound geometric distributions play an important role in reliability theory, queuing theory, and regenerative processes. For

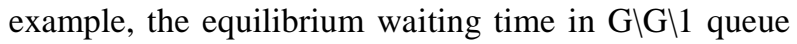
has a compound geometric distribution and so does the maximal aggregate loss of surplus process under the classical and renewal risk models. For more details, Gertsbakh $^{[4-6,10]}$. Mixture of exponential distribution is the only one of the most important distributions in reliability theory because of their properties to distribution function which given in Feller ${ }^{[3]}$. Brawn ${ }^{[1]}$ discussed moment based bounds on the tial of compound geometric distributions. Drozdenko ${ }^{[2]}$ studied some examples of generalizations of mixtures of exponential distributions. Ma and King ${ }^{[8]}$ discussed the calculation of the $n$-fold convolution of generalized exponential-sum distribution functions. But here we discuss the random sum of mixtures of sum of bivariate exponential random variables.
In this study, we consider the distributions of $R=X+Y$ and $W=X /(X+Y)$ when $\mathrm{X}$ and $\mathrm{Y}$ are correlated exponential random variables with the joint pdf given by:

$$
f(x, y)=\left\{\begin{array}{c}
\frac{\lambda(1-a)}{b-a} \exp (-\lambda x) \\
\text { if } x=b y, \\
\frac{\lambda^{2} b(b-1)(1-a)}{(b-a)^{2}} \\
x \exp \left\{-\frac{\lambda(b-1) x+\lambda b(1-a) y}{b-a}\right\} \\
\text { if ay<x<by, } \\
\frac{\lambda(b-1)}{b-a} \exp \left(-\frac{\lambda x}{a}\right) \\
\text { if } x=a y,
\end{array}\right.
$$

for $x, y, \lambda>0$ and $0<a<1<b$.

If $\lambda=1$ in relation (1) we obtain the distribution of Lawrance and Lewis`s bivariate exponential distribution $^{[9]}$.

The research is organized as follow. First, we derive the exact expressions for the pdfs of $R=X+Y$ and $W=X /(X+Y)$. The pdf of mixture of sum of bivariate exponential distribution is obtained. Results give the pdf of the random sum of mixture of sum of bivariate exponential random variables. Finally, conclusion is given. 


\section{MATERIALS AND METHODS}

The distributions of $\mathbf{R}$ and $\mathbf{W}$ : Theorems 1 and 2 derive the pdfs of $R=X+Y$ and $W=X /(X+Y)$ when $\mathrm{X}$ and $\mathrm{Y}$ are distributed according to (1).

Theorem 1: If $\mathrm{X}$ and $\mathrm{Y}$ are jointly distributed according to (1) then:

$$
\begin{aligned}
\mathrm{f}_{\mathrm{R}}(\mathrm{r})= & \frac{\lambda(1-\mathrm{a}) \mathrm{r}}{\mathrm{b}-\mathrm{a}} \exp \left\{-\frac{\lambda \mathrm{rb}}{\mathrm{b}+1}\right\}+\frac{\lambda(\mathrm{b}-1) \mathrm{r}}{\mathrm{b}-\mathrm{a}} \exp \left\{-\frac{\lambda \mathrm{r}}{\mathrm{a}+1}\right\} \\
& -\frac{\lambda \mathrm{b}(\mathrm{b}-1)(1-\mathrm{a})}{(\mathrm{b}-\mathrm{a})(\mathrm{ab}-1)}\left\{\exp \left\{-\frac{\lambda \mathrm{br}}{\mathrm{b}+1}\right\}-\exp \left\{-\frac{\lambda \mathrm{r}}{\mathrm{a}+1}\right\}\right\}
\end{aligned}
$$

Proof: The result immediately follows by elementary integration $^{[9]}$.

Theorem 2: If $\mathrm{X}$ and $\mathrm{Y}$ are jointly distributed according to (1) then:

$$
f_{W}(w)=\left\{\begin{array}{l}
\frac{1-a}{\lambda(b-a) w^{2}} \quad \text { if } w=\frac{b}{b+1}, \\
\frac{\lambda^{2} b(b-a)(1-a)}{\{\lambda[(b-1) w+b(1-a)(1-w)]\}^{2}} \\
\text { if } \frac{a}{a+1}<w<\frac{b}{b+1}, \\
\frac{(b-1) a^{2}}{(b-a) \lambda w^{2}} \quad \text { if } w=\frac{a}{a+1},
\end{array}\right.
$$

for $0<w<1$.

Proof: Nadarajah and $\mathrm{Ali}^{[9]}$.

Mixture of sum of bivariate exponential distributions: We derive the mixture of the distribution of sums $R=X+Y$.

A random variable $X$ has a mixture of distributions if one or more parameters of the distribution of $\mathrm{X}$ is also random variable. Let $\mathrm{g}(\mathrm{x}, \mathrm{y})$ be probability function (for simplicity $\mathrm{pf}$ ) of $\mathrm{X}$, where $\mathrm{y}$ is a parameter of distribution of $\mathrm{X}$. If $\mathrm{y}$ is a value of a random variable $\mathrm{Y}$, then $\mathrm{X}$ has a mixture of distributions. The random variable $Y$ may take continuous distribution with probability density function (pdf) h (y), then the pf of $\mathrm{X}$ :

$f(x)=\int_{-\infty}^{\infty} g(x ; y) h(y) d y$.
The random variable $\mathrm{Y}$ may take discrete distribution with probability $\mathrm{h}(\mathrm{y})$, thus the pf of $\mathrm{X}$ becomes:

$f(x)=\sum_{y=-\infty}^{\infty} g(x ; y) h(y)$

By using Eq. 2 and the definition of mixture of distribution we have:

$$
\begin{aligned}
g(r) & =\int_{0}^{\infty}\left\{\frac{\lambda(1-a) r}{b-a} \exp \left\{-\frac{\lambda b r}{b+1}\right\}+\frac{\lambda(b-1) r}{b-a} \exp \left\{-\frac{\lambda r}{a+1}\right\}\right. \\
& \left.-\frac{\lambda b(b-1)(1-a)}{(b-a)(a b-1)}\left[\exp \left\{-\frac{\lambda b r}{b+1}\right\}-\exp \left\{-\frac{\lambda r}{a+1}\right\}\right]\right\} \\
& \times \frac{\beta(\beta \lambda)^{n-1}}{\Gamma(n)} e^{-\beta \lambda} d \lambda
\end{aligned}
$$

Remark 1: The relation (6) is a general case of a mixture of sum of bivariate exponential distribution (i.e., we are not substituting the value of the distribution of the parameter $\lambda$ ). When we use a certain probability distribution as the probability distribution of the parameter $\lambda$, we find that the values taken by the random variable in such distribution must be in the domain of the possible values for the parameter $\lambda$ (i.e., all the value belong to the parameter space for the main distribution).

\section{RESULTS AND DISCUSSION}

Random sum of mixtures of sum of bivariate exponential distributions: We derive the pdf of random sum of mixtures of sum of bivariate exponential distributions. Let $X_{i}, i=1,2, \ldots, N$ be independent and identically distributed random variables. Each $X_{i}, i=1,2, \ldots, N$ has a mixture of sum of bivariate exponential distribution with pdf as given by using Eq. 4 as follow:

$$
\begin{aligned}
\mathrm{f}_{\mathrm{x}_{\mathrm{i}}}\left(\mathrm{x}_{\mathrm{i}}\right)= & \int_{0}^{\infty}\left\{\frac{\lambda(1-\mathrm{a}) \mathrm{r}}{\mathrm{b}-\mathrm{a}} \mathrm{x}_{\mathrm{i}} \exp \left\{-\frac{\lambda b \mathrm{x}_{\mathrm{i}}}{\mathrm{b}+1}\right\}+\frac{\lambda(\mathrm{b}-1) \mathrm{x}_{\mathrm{i}}}{\mathrm{b}-\mathrm{a}}\right. \\
& \exp \left\{-\frac{\lambda \mathrm{x}_{\mathrm{i}}}{\mathrm{a}+1}\right\}-\frac{\lambda \mathrm{b}(\mathrm{b}-1)(1-\mathrm{a})}{(\mathrm{b}-\mathrm{a})(\mathrm{ab}-1)}\left[\exp \left\{-\frac{\lambda b \mathrm{x}_{\mathrm{i}}}{\mathrm{a}+1}\right\}\right. \\
& \left.\left.-\exp \left\{-\frac{\lambda \mathrm{x}_{\mathrm{i}}}{\mathrm{a}+1}\right\}\right]\right\} \times \frac{\beta(\beta \lambda)^{\mathrm{n}-1}}{\Gamma(\mathrm{n})} \mathrm{e}^{-\beta \lambda} \mathrm{d} \lambda
\end{aligned}
$$

The Laplace transform of this pdf given as follow: 


$$
\begin{aligned}
\mathrm{f}_{\mathrm{x}_{i}}^{*}(\mathrm{~s})= & \mathrm{E}\left[\mathrm{e}^{-s \mathrm{x}_{\mathrm{i}}}\right]=\mathrm{E}\left[\mathrm{f}_{\mathrm{x}_{i} / \lambda}^{*}(\mathrm{~s})\right] \\
& =\mathrm{E}\left[\int_{0}^{\infty} \mathrm{e}^{-s \mathrm{x}_{i}} \frac{\lambda(1-\mathrm{a})}{\mathrm{b}-\mathrm{a}} \times \exp \left\{-\frac{\lambda b x_{i}}{\mathrm{~b}+1}\right\} \mathrm{dx_{i }}\right. \\
& \times+\int_{0}^{\infty} \mathrm{e}^{-s \mathrm{x}_{i}} \frac{\lambda(\mathrm{b}-1)}{\mathrm{b}-\mathrm{a}} \times \exp \left\{-\frac{\lambda b x_{i}}{\mathrm{a}+1}\right\} \mathrm{dx} \mathrm{x}_{\mathrm{i}} \\
& -\int_{0}^{\infty}\left\{\mathrm{e}^{-s \mathrm{x}_{i}} \frac{\lambda \mathrm{b}(\mathrm{b}-1)(1-\mathrm{a})}{(\mathrm{b}-\mathrm{a})(\mathrm{ab}-1)}\right. \\
& \left.\left.\times\left[\exp \left\{-\frac{\lambda b x_{i}}{\mathrm{~b}+1}\right\}-\exp \left\{-\frac{\lambda \mathrm{x}_{\mathrm{i}}}{\mathrm{a}+1}\right\}\right] \mathrm{dx_{i }}\right\}\right]
\end{aligned}
$$

after evaluate above integral in Eq. 8 we have:

$$
\begin{aligned}
\mathrm{f}_{\mathrm{x}_{\mathrm{i}}}^{*}(\mathrm{~s})= & \mathrm{E}\left[\frac{\lambda(1-\mathrm{a})}{\mathrm{b}-\mathrm{a}}\left(\mathrm{s}+\frac{\lambda \mathrm{b}}{\mathrm{b}+1}\right)^{-2}+\frac{\lambda(\mathrm{b}-1)}{\mathrm{b}-\mathrm{a}}\left(\mathrm{s}+\frac{\lambda \mathrm{b}}{\mathrm{a}+1}\right)^{-2}\right. \\
& -\frac{\lambda \mathrm{b}(\mathrm{b}-1)(1-\mathrm{a})}{(\mathrm{b}-\mathrm{a})(\mathrm{ab}-1)}\left[\left[\left(\mathrm{s}+\frac{\lambda \mathrm{b}}{\mathrm{b}+1}\right)^{-1}-\left(\mathrm{s}+\frac{\lambda}{\mathrm{a}+1}\right)^{-1}\right]\right] \\
= & \frac{1-\mathrm{a}}{\mathrm{b}-\mathrm{a}} \int_{0}^{\infty} \lambda\left(\mathrm{s}+\frac{\mathrm{b} \lambda}{\mathrm{b}+1}\right)^{-2} \mathrm{~h}(\lambda) \mathrm{d} \lambda+\frac{\mathrm{b}-1}{\mathrm{~b}-\mathrm{a}} \int_{0}^{\infty} \lambda\left(\mathrm{s}+\frac{\lambda}{\mathrm{a}+1}\right)^{-2} \\
& \mathrm{~h}(\lambda) \mathrm{d} \lambda-\frac{\mathrm{b}(\mathrm{b}-1)(1-\mathrm{a})}{(\mathrm{b}-\mathrm{a})(\mathrm{ab}-1)}\left\{\int_{0}^{\infty} \lambda\left(\mathrm{s}+\frac{\lambda \mathrm{b}}{\mathrm{a}+1}\right)^{-1}\right. \\
& \times \mathrm{h}(\lambda) \mathrm{d} \lambda-\int_{0}^{\infty} \lambda\left(\mathrm{s}+\frac{\lambda}{\mathrm{a}+1}\right)^{-1} \mathrm{~h}(\lambda) \mathrm{d} \lambda
\end{aligned}
$$

Then, the Laplace transform is:

$$
\begin{aligned}
f_{x_{i}}^{*}(s)= & \frac{(1-a)}{(b-a)} \omega+\frac{b-1}{b-a} \psi \\
& -\frac{b(b-1)(1-a)}{(b-a)(a b-1)}\{\vartheta-\rho\},
\end{aligned}
$$

Where

$$
\begin{aligned}
& \omega=\sum_{l=1}^{\infty}(-1)^{l-1} A^{l-1} s^{-l-1} l E\left(\lambda^{l}\right), \\
& \psi=\sum_{l=1}^{\infty}(-1)^{l-1} B^{l-1} s^{-l-1} l E\left(\lambda^{l}\right), \\
& \vartheta=\sum_{k=1}^{\infty}(-1)^{k-1} C^{k-1} s^{-k} E\left(\lambda^{k}\right), \\
& \rho=\sum_{k=1}^{\infty}(-1)^{k-1} D^{k-1} s^{-k} E\left(\lambda^{k}\right) .
\end{aligned}
$$

Assume the random sum $S_{N}=\sum_{i=1}^{N} X_{i}, S_{0}=0, N$ is a non-negative integer valued random variable with $\mathrm{pf}$ $\mathrm{g}(\mathrm{n})=\operatorname{Pr}\{\mathrm{N}=\mathrm{n}\}$. The Laplace transform of the pdf of random sum of mixture of sum of bivariate exponential distributions is given by the following relation:

$$
\mathrm{L}\left\{\mathrm{f}_{\mathrm{S}_{\mathrm{N}}}^{*}(\mathrm{t})\right\}=\mathrm{f}_{\mathrm{S}_{\mathrm{N}}}^{*}(\mathrm{~s})=\mathrm{P}_{\mathrm{N}}\left\{\mathrm{f}_{\mathrm{X}_{\mathrm{i}}^{*}}^{*}(\mathrm{~s})\right\}
$$

where, $P_{N}(\theta)$ is the probability generating function (pgf) of the random variable $\mathrm{N}$. From relations (9) and (10) we get:

$$
\begin{aligned}
& f_{S_{N}}^{*}(s)=\sum_{n=0}^{\infty}\left\{\left[\frac{1-a}{b-a} \omega+\frac{b-1}{b-a} \psi\right]\right. \\
& \left.-\frac{b(b-1)(1-a)}{(b-a)(a b-1)}[\vartheta-\rho]\right\}^{n} g(n),
\end{aligned}
$$

where, $\mathrm{A}=\frac{\mathrm{a}}{\mathrm{b}+1}, \mathrm{~B}=\frac{1}{\mathrm{a}+1}, \mathrm{C}=\frac{\mathrm{b}}{\mathrm{b}+1}$ and $\mathrm{D}=\frac{1}{\mathrm{a}+1}$.

Thus relation (11) becomes:

$\mathrm{f}_{\mathrm{S}_{N}}^{*}(\mathrm{~s})=\sum_{\mathrm{n}=0}^{\infty}\left\{\sum_{\mathrm{l}=1}^{\infty}\left[\omega_{1}+\psi_{1}\right] \mathrm{s}^{-1-1}-\left[\varphi_{\mathrm{k}}-\rho_{\mathrm{k}}\right] \mathrm{s}-\mathrm{k}\right\}^{\mathrm{n}} \mathrm{g}(\mathrm{n})$

where

$$
\begin{aligned}
& \omega_{l}=\alpha(-1)^{l-1} A^{l-1} l E\left(\lambda^{l}\right), \\
& \vartheta_{l}=\beta(-1)^{l-1} B^{l-1} l E\left(\lambda^{l}\right), \\
& \vartheta_{k}=\gamma(-1)^{k-1} C^{k-1} E\left(\lambda^{k}\right), \\
& \rho_{k}=\gamma(-1)^{k-1} D^{k-1} E\left(\lambda^{k}\right), \\
& \alpha=\frac{1-a}{b-a}, \beta=\frac{b-1}{b-a} \text { and } \gamma=\frac{b(b-1)(1-a)}{(b-a)(a b-1)} .
\end{aligned}
$$

Let:

$$
p=\sum_{l=1}^{\infty}\left\{\omega_{l}+\boldsymbol{v}\right\} s^{-l-1}
$$

and

$$
q=\sum_{k=1}^{\infty}\left\{\vartheta_{k}-\rho_{k}\right\} s^{-k}
$$

Substituting from relations (13) and (14) into relation (12) we get:

$$
f_{S_{N}}^{*}(s)=\sum_{n=0}^{\infty} g(n) \sum_{i=0}^{\infty}\left(\begin{array}{l}
n \\
i
\end{array}\right)(-i)^{i} q^{i} p^{n-i}
$$

where

$$
q^{i}=\left\{\sum_{k=1}^{\infty}\left[\vartheta_{k}-\rho_{k}\right] s^{-k}\right\}^{i} .
$$


By using complete multinomial expansions theorem in relation (16), see $\mathrm{Ma}^{[7]}$, we find:

$$
\begin{aligned}
& \left\{\sum_{k=1}^{\infty}\left[\vartheta_{k}-\rho_{k}\right] s^{-k}\right\}^{i} \\
& =i ! \sum_{p=1}^{M} \sum_{m_{1}=1}^{m-(p-1)} \sum_{m_{2}=m_{1}+1}^{m-(p-2)} \cdots \sum_{m_{p}=m_{p-1}+1}^{m} \times \sum_{i_{1}=1}^{i-(p-1)} \\
& \times \sum_{i_{2}=1}^{i-i_{1}-(p-2)} \sum_{i_{p-1}=1}^{i-i_{1}-i_{2}-\cdots-i_{p-2}-1} \ldots \sum_{i=i_{1}-i_{2}-\cdots-i_{p-1}}^{i_{p}=i-i_{1}-i_{2} \cdots-i_{p-1}} \\
& \times \prod_{k=1}^{p} \frac{\left\{\vartheta_{m_{k}}-\rho_{m_{k}}\right\}^{i_{k}}}{i_{k} !} \times \prod_{k=1}^{p} \frac{1}{s^{i_{k} m_{k}}},
\end{aligned}
$$

where

$$
\begin{gathered}
\vartheta_{m_{k}}=\gamma(-1)^{m_{k}-1} C^{m_{k}-1} E\left(\lambda^{m_{k}}\right), \\
\rho_{m_{k}}=\gamma(-1)^{m_{k}-1} D^{m_{k}-1} E\left(\lambda^{m_{k}}\right), \\
M= \begin{cases}i & i<m \\
m & i \geq m\end{cases}
\end{gathered}
$$

Consequently from relation (17) we have:

$$
\begin{aligned}
& \left\{\sum_{k=1}^{\infty}\left[\vartheta_{k}-\rho_{k}\right] s^{-k}\right\}^{i} \\
= & \lim _{m \rightarrow \infty} \sum_{k=1}^{m}\left\{\left\{\vartheta_{k}-\rho_{k}\right\} s^{-k}\right\}^{i} \\
= & i ! \sum_{p=1}^{i} \sum_{m_{1}=1}^{\infty} \sum_{m_{2}=m_{1}+1}^{\infty} \ldots \sum_{m_{p}=m_{p-1}+1}^{\infty} \times \sum_{i_{1}=1}^{i-(p-1)} \\
\times & \sum_{i_{2}=1}^{i-i_{1}-(p-2)} \sum_{i_{p-1}=1}^{i-i_{1}-i_{2}-\cdots-i_{p-2}-1} \ldots \sum_{i-i_{1}-i_{2}-\cdots-i_{p-1}}^{i_{i_{p}=i-i_{1}-i_{2} \cdots-i_{p-1}}} \\
\times & \prod_{k=1}^{p} \frac{\left\{\vartheta_{m_{k}}-\rho_{m_{k}}\right\}^{i_{k}}}{i_{k} !} \times \prod_{k=1}^{p} \frac{1}{s^{i_{k} m_{k}}},
\end{aligned}
$$

and

$$
p^{n-i}=\left\{\sum_{l=1}^{\infty}\left\{\omega_{l}+\not \partial\right\} s^{-l-1}\right\}^{n-i},
$$

also by using the complete multinomial expansions theorem, we find:

$$
\left\{\sum_{l=1}^{u}\left[\omega_{l}+\psi_{l}\right] s^{-l-1}\right\}^{\omega}
$$

$$
\begin{aligned}
& =\omega ! \sum_{r=1}^{U} \sum_{u_{1}=1}^{u-(r-1)} \sum_{u_{2}=u_{1}+1}^{u-(r-2)} \cdots \sum_{u_{r}=u_{r-1}+1}^{u} \times \sum_{\omega_{1}=1}^{\omega-(r-1)} \\
& \times \sum_{\omega_{2}=1}^{\omega-\omega_{1}-(r-2)} \sum_{\omega_{r-1}=1}^{\omega-\omega_{1}-\omega_{2} \cdots-\omega_{p-2}-1} \cdots \sum_{\omega_{l=1}}^{\omega-\omega_{1}-\omega_{2} \cdots-\omega_{r-1}} \\
& \times \prod^{r} \frac{\left\{\omega_{u_{l}}+\psi_{u_{l}}\right\}^{\omega_{l}}}{\omega_{l} !} \times \prod_{k=1}^{p} \frac{1}{s^{\omega_{l} u_{l}+1}}
\end{aligned}
$$

where

$$
\begin{aligned}
& \omega_{u_{l}}=\alpha(-1)^{u_{l}-1} A^{u_{l}-1} u_{l} E\left(\lambda^{u_{l}}\right), \\
& \psi_{u_{l}}=\beta(-1)^{u_{l}-1} B^{u_{l}-1} u_{l} E\left(\lambda^{k}\right), \\
& U=\left\{\begin{array}{ll}
\omega & \omega<u \\
u & \omega \geq u
\end{array}, \omega=n-i,\right.
\end{aligned}
$$

Consequently from relation (19) we have:

$$
\begin{aligned}
& \left\{\sum_{l=1}^{\infty}\left[\omega_{u_{l}}+\psi_{u_{l}}\right] s^{-l-1}\right\}^{\omega} \\
& =\lim _{u \rightarrow \infty}\left\{\sum_{l=1}^{u}\left[\omega_{u_{l}}+\psi_{u_{l}}\right] s^{-l-1}\right\}^{\omega} \\
& =\omega ! \sum_{r=1}^{\infty} \sum_{u_{1}=1}^{\infty} \sum_{u_{2}=u_{1}+1}^{\infty} \cdots \sum_{u_{r}=m_{r-1}+1}^{\infty} \times \sum_{\omega_{l}=1}^{\omega-(r-1)} \\
& \times \sum_{\omega_{2}=1}^{\omega-\omega_{l}-(r-2)} \sum_{\omega_{r-1}=\omega_{l}-\omega_{2} \cdots-\omega_{p-2}-1}^{\omega} \cdots \sum_{\omega_{r}-\omega_{1}-\omega_{2} \cdots-\omega_{r-1}}^{\omega_{r}=\omega-\omega_{l}-\omega_{2}-\cdots \omega_{r-1}} \\
& \times \prod_{l=1}^{r} \frac{\left\{\omega_{u_{l}}+\psi_{u_{l}}\right\}^{\omega_{l}}}{\omega_{l} !} \times \prod_{l=1}^{r} \frac{1}{s^{\omega_{l} u_{l}+1}} .
\end{aligned}
$$

Substituting from relations (19) and (21) into relation (15) we get:

$f_{S_{N}}^{*}(s)=\sum_{n=0}^{\infty} g(n) \sum_{i=0}^{n}\left(\begin{array}{l}n \\ i\end{array}\right)(-1)^{i} \times i ! \psi \Phi$

$\times \prod_{k=1}^{p} \frac{1}{s^{i_{k} m_{k}}} \times \omega ! \Xi \Omega$

$\times \prod_{l=1}^{r} \frac{\left[\omega_{u_{l}}+\boldsymbol{v}_{u_{l}}\right\}^{\omega_{l}}}{\omega_{l} !} \times \prod_{l=1}^{r} \frac{1}{s^{\omega_{l} u_{l}+1}}$,

where

$\psi=\sum_{p=1}^{i} \sum_{m_{1}=1}^{\infty} \sum_{m_{2}=m_{1}+1}^{\infty} \ldots \sum_{m_{p}=m_{p-1}+1}^{\infty}$, 


$$
\begin{aligned}
& \Phi=\sum_{i_{1}=1}^{i-(p-1)} \sum_{i_{2}=1}^{i-i_{1}-(p-2)} \cdots \sum_{i_{p-1}=1}^{i-i_{1}-i_{2} \cdots-i_{p-2}-1} \\
& \times \sum_{i_{p}=i-i_{1}-i_{2}-\cdots-i_{p-1}}^{i-i_{1}-i_{2} \cdots-i_{p-1}}, \\
& \Xi=\sum_{r=1}^{\omega} \sum_{u_{1}=1}^{\infty} \sum_{u_{2}=u_{1}+1}^{\infty} \cdots \sum_{u_{r}=u_{r-1}+1}^{\infty}, \\
& \Omega=\sum_{\omega_{1}=1}^{\omega} \sum_{\omega_{2}=1}^{\omega-(r-1)} \sum_{\omega-\omega_{1}-(r-2)}^{\omega-\omega_{1}-\omega_{2}-\cdots-\omega_{p-2}-1} \ldots \\
& \ldots \sum_{\omega_{r-1}=1}^{\omega-\omega_{1}-\omega_{2}-\cdots-\omega_{r-1}} \ldots \\
& \sum_{r}=\omega-\omega_{1}-\omega_{2}-\cdots-\omega_{r-1}
\end{aligned}
$$

Now we can find the pdf random sum $S_{N}$ by using the inverse Laplace transform of relation (21) as $f_{S_{N}}(t)=L^{-1}\left\{f_{S_{N}}^{*}(s)\right\}$

$=\sum_{n=0}^{\infty} g(n) \sum_{i=0}^{n}\left(\begin{array}{l}n \\ i\end{array}\right)(-1)^{i} \times i ! \psi \Phi$

$\times \prod_{k=1}^{p} \frac{\left\{\vartheta_{m_{k}}-\rho_{m_{k}}\right\}^{i_{k}}}{i_{k} !} \times \omega ! \Xi \Omega$

$\times \prod_{l=1}^{r} \frac{\left\{\omega_{u_{l}}+\psi_{u_{l}}\right\}^{\omega l}}{\omega_{l}}$

$\times L^{-1}\left\{\prod_{k=1}^{p} \frac{1}{s^{i_{k} m_{k}}} \prod_{l=1}^{r} \frac{1}{s^{\omega_{l} u_{l}+1}}\right\}$.

Thus we can write relation (22) as:

$$
\begin{gathered}
f_{S_{N}}(t)=\sum_{n=0}^{\infty} g(n) \sum_{i=0}^{n}\left(\begin{array}{l}
n \\
i
\end{array}\right)(-1)^{i} \times i ! \psi \Phi \\
\times \prod_{k=1}^{p} \frac{\left\{\vartheta_{m_{k}}-\rho_{m_{k}}\right\}^{i_{k}}}{i_{k} !} \times \omega ! \Xi \Omega \\
\times \prod_{l=1}^{r} \frac{\left\{\omega_{u_{l}}+\vartheta_{u_{l}}\right\}^{\omega l}}{\omega_{l}} \\
\times L^{-1}\left\{\frac{1}{\sum_{S^{k=1} i_{k} m_{k}+\sum_{l=1}^{r} \omega_{l} u_{l}+r}}\right\} .
\end{gathered}
$$

Thus the pdf of the random sum $\mathrm{S}_{\mathrm{N}}$ is:

$$
f_{S_{N}}^{*}(s)=\sum_{n=0}^{\infty} g(n) \sum_{i=0}^{n}\left(\begin{array}{l}
n \\
i
\end{array}\right)(-1)^{i} \times i ! \psi \Phi
$$

$$
\begin{aligned}
& \times \prod_{k=1}^{p} \frac{\left\{\vartheta_{m_{k}}-\rho_{m_{k}}\right\}^{i_{k}}}{i_{k} !} \times \omega ! \Xi \Omega \\
& \times \prod_{l=1}^{r} \frac{\left[\omega_{u_{l}}+\psi_{u_{l}}\right\}^{\omega l}}{\omega_{l} !} \times \frac{t^{\mu+\xi-1}}{(\mu+\xi-1) !},
\end{aligned}
$$

where $\mu=\sum_{k=1}^{p} i_{k} m_{k}$ and $\xi=\sum_{l=1}^{r} \omega_{l} u_{l}+r$.

Some important statistical measures: We can obtain the tail probabilities and the failure rate of the random sum $S_{N}$ by using the pdf given in relation (24) as follows:

- Tail probabilities: The tail probabilities of the random sum $S_{N}$ is given by:

$$
\begin{gathered}
a_{t}=\operatorname{Pr}\left(S_{N}>t\right)=1-\operatorname{Pr}\left(S_{N} \leq t\right) \\
=1-\sum_{n=0}^{\infty} g(n) \sum_{i=0}^{n}\left(\begin{array}{c}
n \\
i
\end{array}\right)(-1)^{i} \times i ! \psi \Phi \\
\times \prod_{k=1}^{p} \frac{\left\{\vartheta_{m_{k}}-\rho_{m_{k}}\right\}^{i_{k}}}{i_{k} !} \times \omega ! \Xi \Omega \\
\times \prod_{l=1}^{r} \frac{\left[\omega_{u_{l}}+\psi_{u_{l}}\right\}^{\omega l}}{\omega_{l} !} \times \frac{t^{\mu+\xi}}{(\mu+\xi) !},
\end{gathered}
$$

where $\mu=\sum_{k=1}^{p} i_{k} m_{k}$ and $\xi=\sum_{l=1}^{r} \omega_{l} u_{l}+r$

- Failure rate: The Failure rate of the random sum $S_{N}$ is:

$$
\begin{aligned}
& h_{S_{N}}(t)=\frac{f_{S_{N}}(t)}{1-F_{S_{N}}(t)}=\frac{f_{S_{N}}(t)}{a_{t}} \\
& =\sum_{n=0}^{\infty} g(n) \sum_{i=0}^{n}\left(\begin{array}{c}
n \\
i
\end{array}\right)(-1)^{i} \times i ! \psi \Phi \\
& \times\left[\prod_{k=1}^{p} \frac{\left\{\vartheta_{m_{k}}-\rho_{m_{k}}\right\}^{i_{k}}}{i_{k} !} \times \omega ! \Xi \Omega\right. \\
& \left.\times \prod_{l=1}^{r} \frac{\left[\omega_{u_{l}}+\psi_{u_{l}}\right\}^{\omega l}}{\omega_{l} !} \times \frac{t^{\mu+\xi-1}}{(\mu+\xi-1) !}\right] / \\
& {\left[1-\sum_{n=0}^{\infty} g(n) \sum_{i=0}^{n}\left(\begin{array}{l}
n \\
i
\end{array}\right)(-1)^{i} \times i ! \psi \Phi\right.} \\
& \times \prod_{k=1}^{p} \frac{\left\{\vartheta_{m_{k}}-\rho_{m_{k}}\right\}^{i_{k}}}{i_{k} !} \times \omega ! \Xi \Omega \\
& \left.\times \prod_{l=1}^{r} \frac{\left[\omega_{u_{l}}+\psi_{u_{l}}\right\}^{\omega l}}{\omega_{l} !} \times \frac{t^{\mu+\xi}}{(\mu+\xi) !}\right]
\end{aligned}
$$




\section{CONCLUSION}

In this study we investigate the pdf of random sum of mixtures of sum of bivariate exponential random variables, when the mixing distribution is a continuous, the number of these random variables has a nonnegative discrete distribution. Also, we find the tail probability and failure rate of random sum.

\section{REFERENCES}

1. Brawn, M., 1990. Error bounds for exponential approximations of geometric convolutions. Ann. Probabil., 18: 1388-1402. http://www.jstor.org/stable/2244430

2. Drozdenko, M., and M. Yadrenko, 2003. On some generalizations of mixtures of exponential distributions. http://riskmodels.net/Drozdenko_Yadrenko.pdf

3. Feller, W., 1972. An Introduction To Probability Theory and its Applications. 2nd Edn., John Wiley, New York. ISBN: 978-0-471-25709-7, pp: 704.

4. Gertsbakh, I.B., 1984. Asymptotic methods in reliability theory: A review. Adv. Applied Probabil., $\quad 16$ : 147-175. http://www.jstor.org/pss/1427229

5. Kalashnikov, V., 1994. Topics on Regenerative Processes. CRS Press, Boca Raton, ISBN: 0849386411, pp: 212.
6. Kalashnikov, V., 1997. Geometric Sums Bounds for Rate Events with Applications. Kluwer Academic Publishers, Dordecht, ISBN: 0792346165, pp: 265.

7. Ma, N., 2001. Complete multinomial expansions. Applied Math. Comput., 124: 365-370. http://portal.acm.org/citation.cfm?id=567382

8. Ma, N.Y. and R.P. King, 2003. The n-fold convolution of generalized exponential-sum distribution functions. Applied Math. Comput., 142: 23-33.

http://portal.acm.org/citation.cfm?id=881934

9. Nadarajah, S. and M. Masood Ali, 2006. The distribution of sums, products and ratios for Lawrance and Lewis's bivariate exponential random variables. Comput. Stat. Data Anal., 50: 3449-3463.

http://cat.inist.fr/?aModele=afficheN\&cpsidt=1784 9383

10. Harry Panjer, H. and E. Gordon Willmot, 1992. Insurance Risk Models. Society of Actuaries, Schumburg, ISBN: 0938959255, pp: 442. 